\title{
The effect of periodic bottlenecks on the competitive ability of Drosophila pseudoobscura lines
}

\author{
JOSÉ A. LÓPEZ BUENO, ANDRÉS MOYA \& FERNANDO GONZÁLEZ-CANDELAS \\ Departament de Genètica, Universitat de València, Dr. Moliner, 50, Burjassot, 46100-Valencia, Spain
}

\begin{abstract}
Competition experiments between several Drosophila pseudoobscura strains that were previously subjected to periodic bottlenecks of different sizes and $D$. willistoni have been carried out. Contrary to previous results with these two species, where stable coexistence was detected, populations of $D$. pseudoobscura displaced D. willistoni in a few generations. By using a relative fitness measure, the control lines (that had not gone through bottlenecks) outcompeted D. willistoni faster than bottlenecked lines. One of these, corresponding to the minimum possible bottleneck size of one pair, also shows significantly lower relative fitness than the other strains. These results are discussed in relation to the effect of periodic bottlenecks in reducing genetic variability and its incidence on the competitive ability of these lines.
\end{abstract}

Keywords: bottlenecks, competition, D. pseudoobscura, flush-crash, genetic drift, relative fitness.

\section{Introduction}

Is there any relationship between the genetic variability of a population and its fitness? The effects of fluctuations of population size upon genetic variability maintained in natural populations have been of interest to population geneticists during the last 50 years (Wright, 1938). Especially relevant is the situation in which a population goes through a severe but temporary reduction in size, i.e. a bottleneck. When population sizes reduce, populations loose genetic variability. This loss of variability should be interpreted in terms of a reduced number of alleles (Nei et al., 1975; Maruyama \& Fuerst, 1984, 1985a,b), of a reduced variance in quantitative traits (Franklin, 1980; Falconer, 1989) as well as in a population being susceptible to extinction because of lack of adaptive flexibility (Frankel \& Soulé, 1981; Beardmore, 1983). Nevertheless, several authors have indicated that in models where gene effects (both intra and interlocus) are not additive, the population genetic variance can be increased after a bottleneck (Bryant et al., 1986, Goodnight, 1988). Carson \& Templeton (1984) also suggest this possibility in an antagonistic pleiotropy model. In any case, a bottleneck will have some effect on the genetic variance and the total number of alleles or the heterozygosity. Bottlenecks, and consequently genetic varia-

Correspondence: F. González-Candelas. tion and fitness, appear as key factors in a different context, the founder effect speciation theory, proposed by Carson $(1968,1971)$, where the establishment of a reproductively isolated population is triggered by the passage through a population bottleneck followed by several generations without selection and culminated by a selection phase.

There are several possibilities for the relationship between genetic variability and fitness, but the most plausible are (i) lack of correspondence between genetic variability and fitness, (ii) a linearly increasing relationship between them, and (iii) an asymptotically increasing relation, in which each additional variable locus contributes in a smaller extent to fitness (Frankel \& Soulé, 1981).

Since 1985 , we have been carrying out an experiment to test the founder effect speciation theory proposed by Carson $(1968,1971)$ using a periodic founder-flush-crash protocol (Galiana et al., 1989). This experiment has provided us with the opportunity to study fully the evolutionary biology of periodically bottlenecked flies. Consequently, we are studying several quantitative characters (including morphologic, meristic and life history traits), pre- and postzygotic sexual isolation and electrophoretic polymorphisms, among others.

Intraspecific competition experiments were introduced by Zimmering (1948) as 'a means of determining the relative fitness of different species or strains of the 
same species'. In this work we report on how periodic bottlenecks of different sizes affect the global fitness of several Drosophila pseudoobscura populations, by using a relative fitness measure. We have carried out competition experiments between different $D$. pseudoobscura laboratory populations that had suffered periodic bottlenecks of different sizes and a laboratory strain of $D$. willistoni.

\section{Materials and methods}

\section{'Flush-crash' protocol}

Fourteen $D$. pseudoobscura strains were used in these experiments. Seven strains were derived from a collection in Bryce Canyon National Park (Utah, U.S.A.) and the other seven were derived from one population collected at Lago Zirahuén (Michoacán, Mexico). These localities are about $2,000 \mathrm{~km}$ apart, and the corresponding $D$. pseudoobscura populations show an important difference: the Bryce Canyon $(\mathrm{BC})$ population has a very low degree of inversion polymorphisms in the third chromosome whereas the Lago Zirahuén (M) population is highly polymorphic for third chromosome inversions. One strain of each geographical origin (BCA and MA) has been maintained under standard laboratory conditions, with serial transfer (Mueller \& Ayala, 1981) in $150 \mathrm{ml}$ bottles with $25 \mathrm{ml}$ of food $\left(21^{\circ} \mathrm{C}\right.$ temperature and varying humidity $)$, and a roughly constant number of 250 individuals per bottle. These strains will be referred to as the ancestral strains.

Five strains from each $\mathrm{BC}$ and $\mathrm{M}$ population have been maintained, since 1985, following a periodic population flush-crash experimental protocol (described in detail by Galiana et al., 1989) with bottleneck sizes of one (BC32 and M3), three (BC7 and M7), five (BC13 and M13), seven (BC19 and M19) and nine (BC25 and M25) pairs. After each bottleneck, the strains were allowed to grow exponentially for several generations. The exponential growth was achieved using 10 different culture bottles for each strain in each generation. Each bottle in the next new generation was initiated with 10 pairs of individuals, which has been randomly sampled (one pair from each) from the 10 bottles of the previous generation. This protocol allows an exponential growth for several generations while keeping the effects of genetic drift at a minimum despite the sampling of individuals that give rise to a new generations of exponential growth, as described, each strain underwent an intense larval competition stress, achieved simply by not limiting to 20 the number of individuals used to start each bottle in the following generation. From the adults recovered among the 10 bottles for each strain after this intense competition phase the corresponding number of pairs were randomly selected to continue the culture after the bottleneck. As a whole, these 10 strains will be known as derived strains.

Finally, the remaining strain of each population was subjected to eight consecutive generations of brother by sister matings, resulting in highly endogamic strains (BCE and $\mathrm{ME}$ ), known generically as endogamic strains. After eight generations of endogamic matings, these strains were cultured according to the standard protocol used for the ancestral populations. Figure 1 shows a plot of population numbers versus time for one strain of each type. The competition experiments were carried out with derived lines that had already gone through nine complete flush-crash cycles.

The $D$. willistoni strain used in these experiments was obtained from the Drosophila Culture Stock at Bowling Green (Ohio).

\section{Competition experiments}

Competition cultures $(150 \mathrm{ml}$ bottles with $50 \mathrm{ml}$ of standard corn medium for Drosophila) were started with $20 \mathrm{D}$. willistoni and $20 \mathrm{D}$. pseudoobscura pairs and kept at $21^{\circ} \mathrm{C}$. Five replicates were made for each strain (two ancestral, 10 derived, and two endogamic).

Flies were allowed to lay eggs during 3 days and then they were discarded. Cultures were screened on a daily basis and, after the emergence of the first adult, the number of emerging individuals during 2 weeks

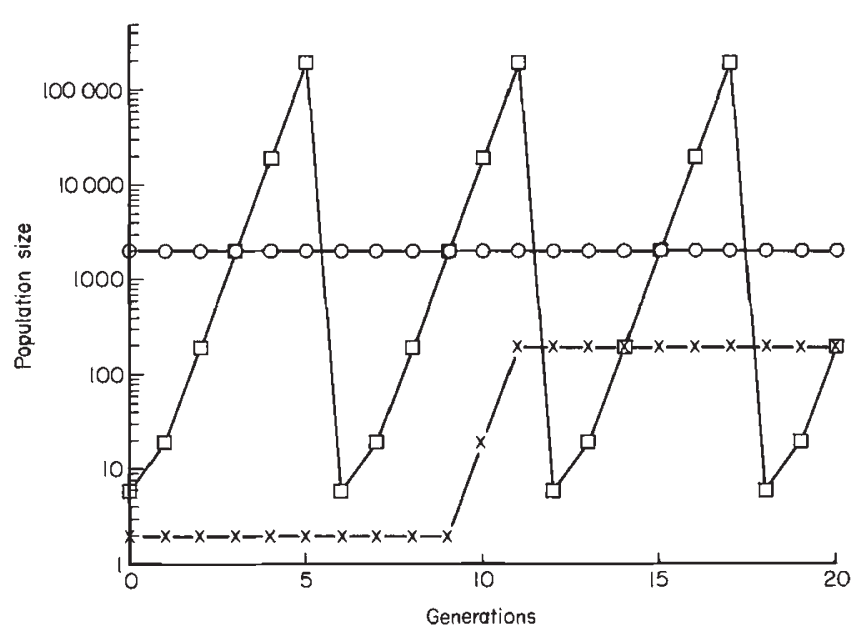

Fig. 1 Variation of population sizes for the three different types of populations $((0)$ ancestral, $(\square)$ derived, and $(*)$ endogamic) during three flush-crash cycles of the derived populations. The competition stress in the derived populations takes place at their highest population sizes, just prior to the bottleneck. 
was scored. Flies emerging between days 5 and 12 after the first one were used to establish a new bottle to continue competition. This time interval was chosen in order to keep the 70 different competition experiments synchronized, so that environmental effects for the different strains and replicates of the same strain were minimized. This process was repeated every generation until one species completely displaced the other.

\section{Results}

All $D$. pseudoobscura lines were very efficient competitors of $D$. willistoni. Complete displacement of $D$. willistoni took place between generations three and five after the onset of competition (Table 1). For both $\mathrm{M}$ and $\mathrm{BC}$ populations, the ancestral lines were the best competitors, as it only took them two generations to displace $D$. willistoni completely.

Table 2 shows the number of individuals recovered as adults after each generation of competition for both species. It must be stressed that only those individuals recovered between days 5 and 12 were used to establish the next generation. These data are shown in Table 3.

In order to compare the relative competitive abilities of the D. pseudoobscura lines, we have worked with the following variable

$y=-\ln [(T D w / D w) /(T D p / D p)]$,

where $T D w$ represents the total number of $D$. willistoni adults recovered in a given generation started with $D w$ $D$. willistoni individuals, and equivalently for $T D p$ and $D p$. The regression coefficient of $y$ versus generation is taken as a measure of the fitness of the corresponding D. psdeudoobscura experimental population.

The regression analyses (Table 4) show that all the slopes are significantly different from zero. A zerovalued slope would correspond to the situation of equal competitive ability between both competitors; the higher a slope the better competitor the corresponding population is. Hence, comparisons among the different populations have been based on comparisons among slopes.

There are several hypotheses that can be tested. Firstly, is there any difference in the competitive abilities of the D. pseudoobscura strains used in the study? In other words, are the 14 slopes homogeneous or not? The analysis of covariance for all the slopes shows that they are nearly homogeneous $(F=1.63$ with 13 and 176 d.f., $0.05<P<0.10)$. From the data in Table 4, it seems that for both groups the ancestral populations are better competitors and that BC32 is a worse competitor than the rest. This has been tested by means of partial $t$-tests with respect to the common slope $(b=0.8530)$, with significant differences in all cases $(t=-3.17, P<0.01$ for BC32, $t=2.04, P<0.05$ for BCA, and $t=1.98, P<0.05$ for MA). After correction for multiple simultaneous comparisons using Bonferroni's inequality, only BC 32 shows a significant, lower slope than the remaining strains.

Secondly, divergent results are obtained when both groups of strains, depending on their origin, are tested separately $(F=2.53$ with 6 and 90 d.f., $P<0.05$ for BC populations, and $F=0.89$ with 6 and 86 d.f., $P>0.50$ for $\mathrm{M}$ populations). Hence, the lines derived from Bryce Canyon can be considered to have non-homo-

Table 1 Number of replicates showing $D$. willistoni individuals at the beginning of the corresponding generation for each competition experiment. The number between parentheses after each strain corresponds to the number of pairs in the corresponding bottlenecks

\begin{tabular}{|c|c|c|c|c|c|c|c|}
\hline \multirow[b]{2}{*}{ Generation } & \multicolumn{7}{|l|}{ Strain } \\
\hline & $\mathrm{BCE}$ & $\mathrm{BC} 32(1)$ & $\mathrm{BC} 7(3)$ & $\mathrm{BC} 13(5)$ & $\mathrm{BC} 19(7)$ & $\mathrm{BC} 25(9)$ & $\mathrm{BCA}$ \\
\hline 1 & 5 & 5 & 5 & 5 & 5 & 5 & 5 \\
\hline 2 & 5 & 5 & 5 & 5 & 5 & 5 & 5 \\
\hline 3 & 4 & 5 & 5 & 1 & 3 & 5 & - \\
\hline \multirow[t]{2}{*}{4} & 2 & 1 & - & - & 1 & - & - \\
\hline & $\mathrm{ME}$ & M3 (1) & $\mathrm{M} 7(3)$ & $\operatorname{M13(5)}$ & $\operatorname{M19}(7)$ & $\mathrm{M} 25(9)$ & MA \\
\hline 1 & 5 & 5 & 5 & 5 & 5 & 5 & 5 \\
\hline 2 & 5 & 5 & 4 & 5 & 4 & 5 & 4 \\
\hline 3 & 4 & 2 & 4 & 4 & 3 & 4 & - \\
\hline 4 & 1 & - & 2 & 2 & - & - & - \\
\hline
\end{tabular}


Table 2 Average number of individuals, with standard error, at the end of each generation of competition of $D$. willistoni $(D w)$ and the corresponding $D$. pseudoobscura $(D p)$ strains. Only cultures with $D$. willistoni individuals (see Table 1$)$ have been considered

\begin{tabular}{|c|c|c|c|c|c|c|c|c|}
\hline \multirow[b]{3}{*}{ Strain } & \multicolumn{8}{|l|}{ Generation } \\
\hline & \multicolumn{2}{|l|}{1} & \multicolumn{2}{|l|}{2} & \multicolumn{2}{|l|}{3} & \multicolumn{2}{|l|}{4} \\
\hline & $D p$ & $D w$ & $D p$ & $D w$ & $D p$ & $D w$ & $D p$ & $D w$ \\
\hline $\mathrm{BCE}$ & $883.8 \pm 51.2$ & $204.0 \pm 22.8$ & $454.8 \pm 40.4$ & $83.4 \pm 26.3$ & $409.5 \pm 82.8$ & $9.3 \pm 5.3$ & $454.5 \pm 49.5$ & $4.0 \pm 1.0$ \\
\hline $\mathrm{BC} 13$ & $322.6 \pm 57.8$ & $34.6 \pm 7.1$ & $551.6 \pm 48.3$ & $3.8 \pm 0.7$ & $406.7 \pm 57.9$ & $1.7 \pm 1.7$ & & \\
\hline BC19 & $65.6 \pm 17.4$ & $86.0 \pm 7.3$ & $517.0 \pm 99.2$ & $122.6 \pm 76.6$ & $435.8 \pm 51.8$ & $46.4 \pm 41.2$ & & \\
\hline $\mathrm{BC} 25$ & $23.0 \pm 0.7$ & $207.2 \pm 8.7$ & $427.4 \pm 52.6$ & $242.8 \pm 56.8$ & $479.2 \pm 36.7$ & $54.8 \pm 15.9$ & $410.5 \pm 82.9$ & $0.0 \pm 0.0$ \\
\hline $\mathrm{BCA}$ & $483.0 \pm 46.7$ & $54.6 \pm 10.3$ & $627.0 \pm 40.4$ & $7.6 \pm 2.2$ & & & & \\
\hline $\mathrm{ME}$ & $644.4 \pm 76.9$ & $293.0 \pm 25.8$ & $655.4 \pm 93.7$ & $54.8 \pm 22.1$ & $356.8 \pm 71.2$ & $5.8 \pm 2.5$ & $475.0 \pm 131.0$ & $0.5 \pm 0.5$ \\
\hline M3 & $298.2 \pm 34.7$ & $38.8 \pm 11.1$ & $445.6 \pm 62.7$ & $7.4 \pm 2.6$ & $313.3 \pm 62.9$ & $0.7 \pm 0.3$ & & \\
\hline M25 & $153.8 \pm 32.0$ & $112.8 \pm 21.5$ & $599.2 \pm 98.2$ & $67.8 \pm 23.7$ & $412.4 \pm 64.7$ & $15.6 \pm 7.0$ & & \\
\hline $\mathrm{MA}$ & $461.6 \pm 101.1$ & $92.2 \pm 24.8$ & $522.5 \pm 69.8$ & $6.8 \pm 2.7$ & & & & \\
\hline
\end{tabular}

Table 3 The average number of individuals, with standard error, at the start of each generation of competition of $D$. willistoni $(D w)$ and the corresponding $D$. pseudoobscura $(D p)$ strains. Only cultures with $D$. willistoni individuals (see Table 1 ) have been considered

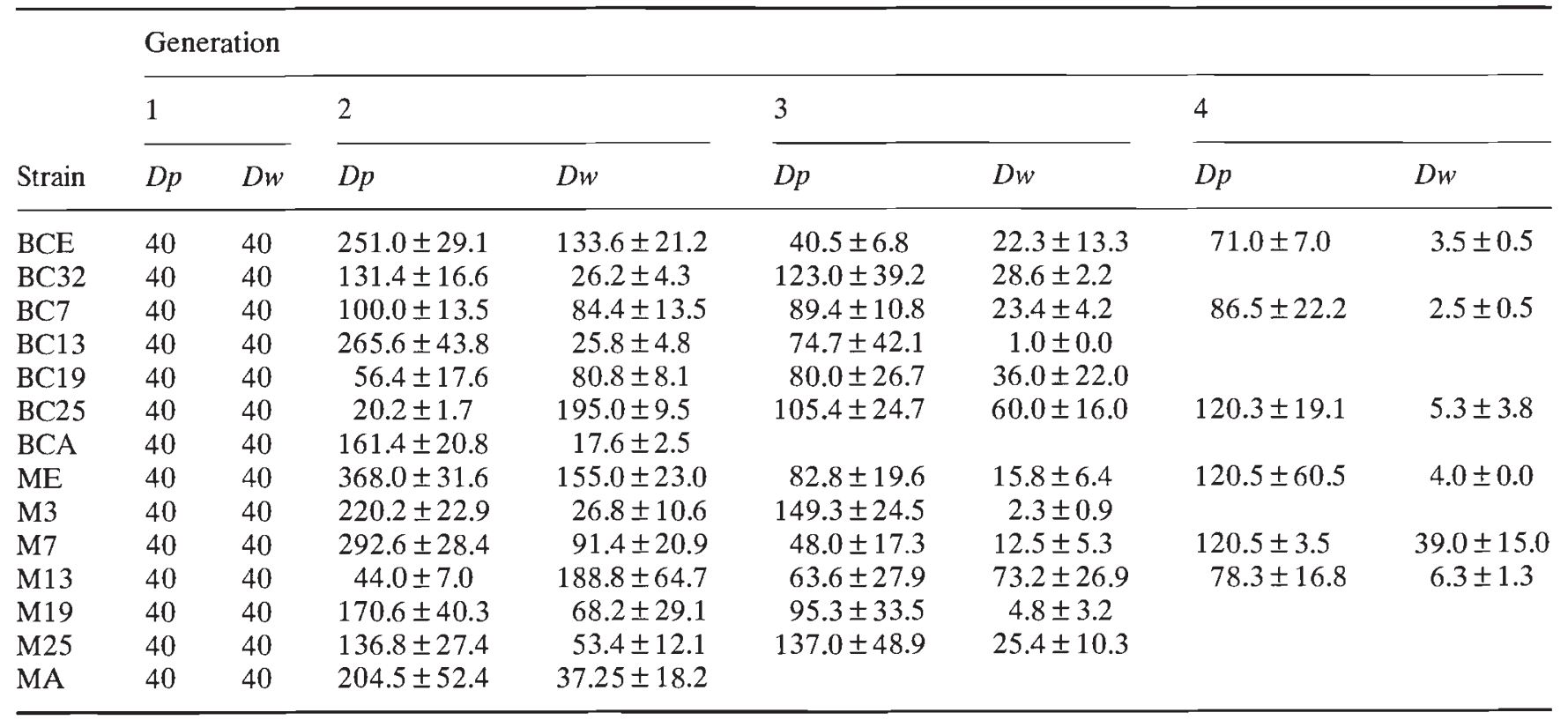

geneous competitive abilities whereas this is not true for the Mexican ones. Thirdly, we are interested in checking the effect of genome organization of the initial population on the evolution of competitive ability. This has been done by testing the equality of the 14 slopes grouped by bottleneck size. Of the seven pairwise tests, only the comparison between BC 32 and M3 (bottleneck size equal to one couple) is significant $(F=9.29$ with 1 and 26 d.f., $P<0.01$ ) even after correction for multiple comparisons.

\section{Discussion}

Laboratory competition experiments between different Drosophila species (reviewed in Arthur, 1982 and Barker, 1983) have demonstrated both the possibility 

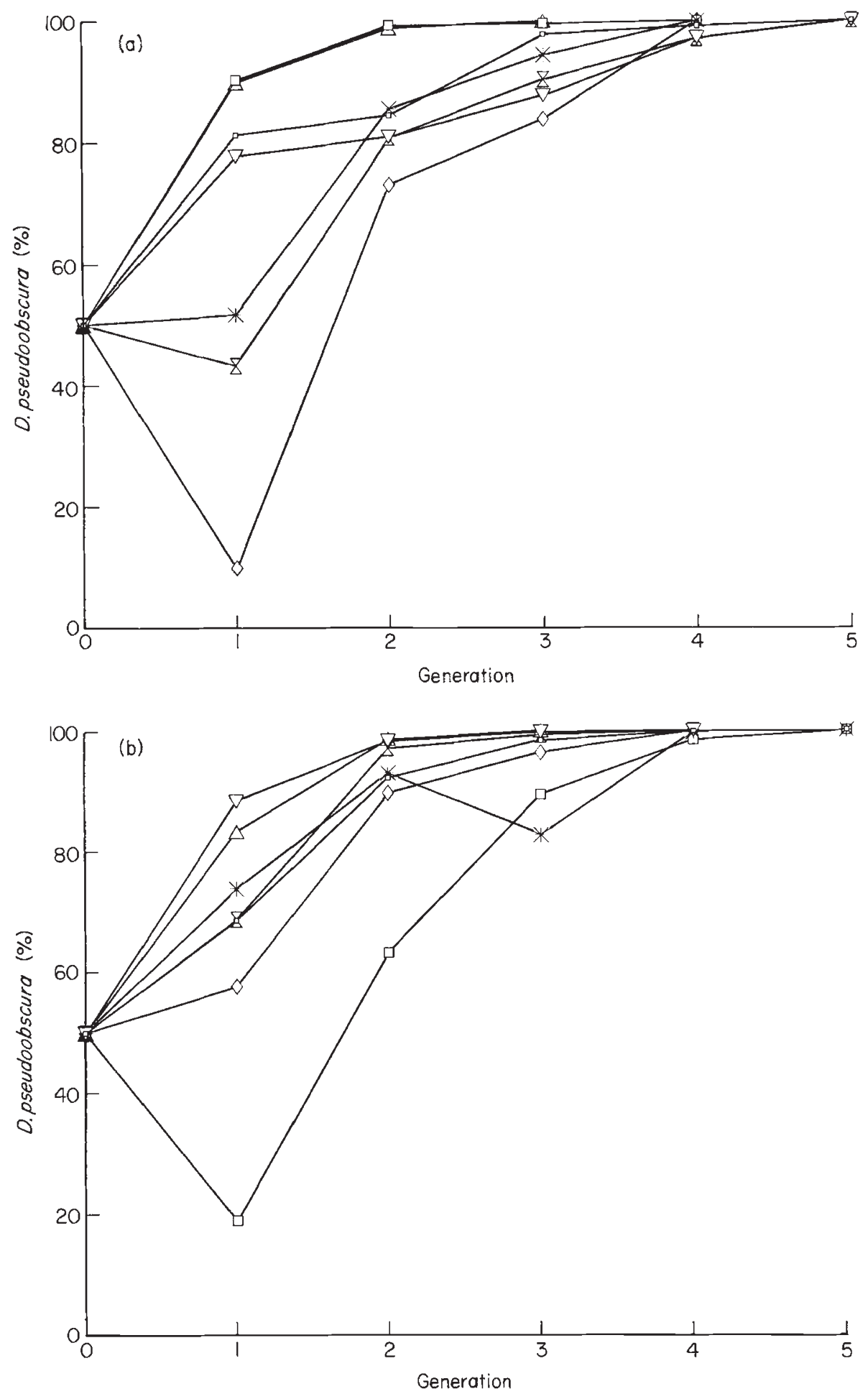

Fig. 2 Relative frequency of adults of the different $D$. pseudoobscura strains at the start of each generation during the competition with $D$. willistoni. (a) BC lines $(\square) \mathrm{BCE},(\nabla) \mathrm{BC} 32,(*) \mathrm{BC} 7$, (ㅁ) BC13, ( $\left.\frac{\nabla}{\Delta}\right) \mathrm{BC} 19,(\diamond) \mathrm{BC} 25,(\Delta)$ $\mathrm{BCA}$; (b) M lines () ME, ( $\nabla) \mathrm{M} 3$,

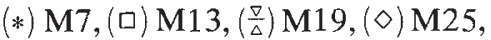
$(\triangle)$ MA. The values represented correspond to the average of remaining replicates at the beginning of each generation (see Table 1).

of a stable coexistence between species (Ayala, 1971 and Ayala et al., 1973) and of a more or less rapid displacement of one competitor by the other (Zimmering, 1948; Ayala, 1965, 1966, 1969). Similar competition experiments have been used to evaluate the relative fitness of different strains of the same species (Zimmer- ing, 1948; Goldstein, 1953; Shugart \& Blaylock, 1973; Mueller et al., 1991), under the assumption of a strong positive correlation between competitive ability and fitness (Zimmering, 1948).

In the case of competition between D. pseudoobscura and D. willistoni, Ayala (1971) obtained a 
Table 4 Regression coefficients, and their corresponding standard errors, for the relative proportion of $D$. williston $i$ individuals to $D$. pseudoobscura $\{\log$ transformed, $y=-\ln$ $[T D w / D w) /(T D p / D p)]\}$ versus generation. All slopes have been tested to be different from zero

\begin{tabular}{llll}
\hline Strain & Bottleneck size & Slope & Standard error \\
\hline BE & Endogamic & $0.8147^{* * *}$ & 0.1335 \\
BC32 & 1 couple & $0.3736^{*}$ & 0.1326 \\
BC7 & 3 couples & $0.6224^{* * *}$ & 0.0830 \\
BC13 & 5 couples & $1.1499^{* *}$ & 0.2502 \\
BC19 & 7 couples & $0.7854^{* * *}$ & 0.1488 \\
BC25 & 9 couples & $0.6378^{*}$ & 0.2393 \\
BCA & Ancestral & $1.3982^{* * *}$ & 0.1655 \\
ME & Endogamic & $0.7536^{* * *}$ & 0.0835 \\
M3 & 1 couple & $0.9673^{* * *}$ & 0.1710 \\
M7 & 3 couples & $0.7701^{* * *}$ & 0.1306 \\
M13 & 5 couples & $0.7083^{* *}$ & 0.1928 \\
M19 & 7 couples & $0.7962^{* * *}$ & 0.1769 \\
M25 & 9 couples & $0.7356^{* * *}$ & 0.1605 \\
MA & Ancestral & $1.4288^{* *}$ & 0.2870 \\
\hline
\end{tabular}

${ }^{*} P<0.05 ; * * P<0.01,{ }^{* * *} P<0.001$.

frequency-dependent equilibrium at $21.5^{\circ} \mathrm{C}$ after several generations of competition. In fact, we decided to choose this temperature to facilitate a competition process able to attain stability after several generations. Unfortunately this was not the case and within three to five generations $D$. willistoni was completely eliminated. We do not know the factors responsible for this rapid displacement or competitive exclusion but, in any case, the exclusion results are not completely homogeneous for the different $D$. pseudoobscura populations.

Following Carson (1961), the relative fitness of a population can be evaluated by the average population size when the population has achieved an equilibrium by natural selection after several generations in a given environment. According to this definition, it is evident that the derived populations perform worse than the ancestral ones in these competition experiments. This is probably due to the loss by drift of alleles favourable for competition, and that consequently confer a higher fitness. Although once the competition was over the populations were not allowed to reach an equilibrium, the average number of individuals at the end of the competition was higher in the ancestral lines than in the derived ones (Table 2, $F=9.238$ with 1 and 68 d.f., $P<0.01$ ). A similar relationship between competitive ability and genetic variability was found by Shugart \& Blaylock (1973) when D. melanogaster was set to compete with $D$. simulans strains with different degrees of genetic variability. In this case, increased variability was found to increase the competitive ability of the corresponding strain.

Furthermore, there is one case, population $\mathrm{BC} 32$, for which the decrease in competitive ability is highly significant not only with respect to the ancestral populations, but also with respect to the other derived ones. This is a one-pair size bottlenecked population, the most likely to suffer drastic effects from genetic drift. Its reduced fitness can be a reflection of inbreeding depression. However, the other one-pair per bottleneck population, M3, does not show any indication of depression, having the second largest relative competitive ability among the derived lines (Table 4). These two are also the only strains that show significant differences in the pairwise comparisons. This difference could be due either to the fixation, or at least increase to near fixation, of alleles determining a reduced competitive ability in $\mathrm{BC} 32$ or to the differences under this experimental protocol, whichever they might be, derived from the polymorphic third chromosomes in the Mexican lines. But as this is the only case with significant differences between $\mathrm{BC}$ and $\mathrm{M}$ populations in the pairwise comparisons, we think the first possibility is more likely. It is also remarkable that a couple of flush-crash cycles later, clear signs of inbreeding depression in the M3 population were detected (J. A. López-Bueno et al., unpublished observations), which show highly reduced viability and fertility that almost drove this population to extinction, whereas none of these signs has yet appeared in BC32.

According to the theory proposed by Carson (1968, 1971), a genetic architecture that does not allow the genome to become uniform would facilitate the fixation of new variants that ultimately could give rise to a new species. It is known that chromosomal inversions act to reduce the rate of recombination between homologous chromosomes carrying different inversions and, consequently, a population rich in chromosome polymorphisms, such as the M populations in this study, would be more likely to differentiate from the ancestral ones. If this theory is correct, and this awaits empirical corroboration that is being developed in our laboratory, the results reported here indicate that genetic differentiation does not correlate with increased fitness. The derived populations from both geographical origins, and with different degrees of polymorphism for third chromosome inversions, have reduced fitness with respect to the ancestral lines. It is even a contradiction to this hypothesis because BC populations, with a genetic architecture that allows free recombination in third chromosomes, have significantly different competitive abilities whereas $\mathrm{M}$ populations do not. 


\section{Acknowledgements}

We would like to thank two anonymous reviewers for their comments and suggestions. JALB was supported by a fellowship from the Spanish Ministerio de Educación y Ciencia (Formación de Personal Investigador).

\section{References}

ARTHUR, W. 1982. The evolutionary consequences of interspecific competition. Adv. Ecol. Res., 12, 127-187.

AYALA, E. J. 1965. Relative fitness of populations of Drosophila serrata and Drosophila birchii. Genetics, 51, 527-544.

AYALA, F. J. 1966. Dynamics of populations. I. Factors controlling population growth and population size in Drosophila serrata. Am. Nat., 100, 333-344.

AYALA, F. J. 1969. Genetic polymorphism and interspecific competitive ability in Drosophila. Genet. Res., 14, 95-102.

AYALA, F. J. 1971. Competition between species: Frequency dependence. Science, 171, 820-824.

AYAlA, F. J., Gilpin, M. E. AND EHRENFEld, J. G. 1973. Competition between species: Theoretical models and experimental tests. Theor. Pop. Biol., 4, 331-356.

BARKER, J. S. F. 1983. Interspecific competition. In: Ashburner, M., Carson, H. L. and Thompson Jr, J. N. (eds) The Genetics and Biology of Drosophila, vol. 3c. Academic Press, London, pp. 285-341.

BEARDMORE, J. A. 1983. Extinction, survival and genetic variation. In: Schonewald-Cox, C. M., Chambers, S. M., MacBryde, and Thomas, L. (eds) Genetics and Conservation, The Benjamin/Cummings Publishing Company, Inc., pp. $125-151$.

BRYANT, E. H., McCOMMAS, S. A. AND COMBS, L. M. 1986. The effect of an experimental bottleneck upon quantitative genetic variation on the housefly. Genetics, 114, 1191-1211.

CARSON, H. L. 1961. Heterosis and fitness in experimental populations of Drosophila melanogaster. Evolution, 15, 496-509.

CARSON, H. L. 1968. The population flush and its genetic consequences. In: Lewontin, R. C. (ed.) Population Biology and Evolution, Syracuse University Press, Syracuse, New York, pp. 123-137.

CARSON, H.L. 1971. Speciation and the founder principle. Stadler Genet. Symp. Univ. Missouri, 3, 51-70.

CARSON, H. L. AND TEMPLETON, A. R. 1984. Genetic revolutions in relation to speciation phenomena: the founding of new populations. Ann. Rev. Ecol. Syst., 7, 311-346.
FALCONER, D. S. 1989. Introduction to Quantitative Genetics, 3rd edn. Longman Press, Oxford.

FRANKEL, O. H. AND SOULE, M. E. 1981. Conservation and Evolution. Cambridge University Press, Cambridge.

FRANKLIN, I. R. 1980. Evolutionary change in small populations. In: Soulé, M. and Wilcox, B. (ed.), Conservation Biology: An Evolutionary Ecological Perspective, Sinauer Associates, Sunderland, MA, pp. 135-149.

galiana, A., AYAla, F. J. AND MOYA, A. 1989. Flush-crash experiments in Drosophila. In: Fontdevila, A. (ed.) Evolutionary Biology of Transient Unstable Populations, Springer-Verlag, Berlin, pp. 58-73.

GOLDSTEIN, L. 1953. Recherches sur les populations mixtes expérimentales de Drosophila melanogaster et Drosophila simulans. Int. Cong. Genet. Bellagio (Como) Part II, $668-670$.

GOODNiGHT, J. C. 1988. Epistasis and the effect of founder events on the additive genetic variance. Evolution, 39, 1380-1383.

MARUYAMA, T. AND FUerst, P. 1984. Population bottlenecks and non equilibrium models. I. Allele numbers when populations evolve from zero variability. Genetics, 108, $745-763$

MARUYAma, T. AND FUerst, P. 1985a. Population bottlenecks and non equilibrium models. II. Number of alleles in a small population that was formed by a recent bottleneck. Genetics, 111, 675-689.

Maruyama, T. AND FUerst, P. 1985b. Population bottlenecks and non equilibrium models. III. Genic homozygosity in populations which experience periodic bottlenecks. Genetics, 111, 691-703.

MUElleR, L. D. AND AYAlA, F. J. 1981. Dynamics of singlespecies population growth: Experimental and statistical analysis. Theor. Pop. Biol., 20, 101-117.

MUELLER, L. D., GONZÁLEZ-CANDELAS, F. AND SWEET, V. F. 1991. Components of density-dependent population dynamics: models and tests with Drosophila. Am. Nat., 137, 457-475.

NEI, M., MARUYAMA, T. AND CHAKRABORTY, R. 1975. The bottleneck effect and genetic variability in populations. Evolution, 29, 1-10.

SHUGART, H. H., JR AND BLAYLOCK. B. G. 1973. The niche-variation hypothesis: an experimental study with Drosophila populations. Am. Nat., 107, 575-579.

WRIGHT, S. 1938. Size of population and breeding structure in relation to evolution. Science, $\mathbf{8 7}, 430-431$.

ZIMMERING, s. 1948. Competition between Drosophila pseudoobscura and Drosophila melanogaster in population cages. Am. Nat., 82, 326-330. 